\title{
Protective applications of vacuum-deposited perfluoropolymer films
}

\author{
K.P. Grytsenko ${ }^{1, *}$, Yu.V. Kolomzarov ${ }^{1}$, O.E. Belyaev ${ }^{1}$, S. Schrader ${ }^{2}$ \\ ${ }^{1}$ V. Lashkaryov Institute of Semiconductor Physics, National Academy of Sciences of Ukraine, \\ 41, prospect Nauky, 03028 Kyiv, Ukraine \\ *Corresponding author phone/fax: +38(044)-525-55-30; e-mail: d.grytsenko@gmail.com \\ ${ }^{2}$ University of Applied Sciences Wildau, Hochschulring 1, Wildau, 15745, Germany
}

\begin{abstract}
The paper summarizes in brief applications of perfluoropolymer thin films deposited from the gas phase by several different methods, for protection of various materials and devices both at the research stage and already in industry. Perfluoropolymer films protect from corrosion coated metals, attain superhydrophobic properties to various surfaces, enhance the lifetime of medical implants, passivating the surface of semiconductors, protect functional layers in organic light emitting diodes, field effect transistors etc. Organic molecules in the PTFE matrix revealed extreme stability and presents the new material for optical applications.
\end{abstract}

Keywords: perfluoropolymer, polytetrafluoroethylene, thin film, coating, protective, matrix, dye, composite.

Manuscript received 14.01.16; revised version received 30.03.16; accepted for publication 08.06.16; published online 06.07.16.

\section{Introduction}

First scientific researches concerning thin polymer film production by decomposition of bulk polymer in vacuum and condensation of small fragments of macromolecules on substrate surface were made in mid- $20^{\text {th }}$ century. Polytetrafluoroethylene (PTFE) and polyhexafluoropropylene were among the first tested polymers. Different perflouoropolymer (PFP) films were deposited using various gas phase methods: thermal decomposition, magnetron sputtering (MSp), laser ablation (LA), plasma polymerisation (PP) and hot-wire chemical vapour deposition (HW CVD). Partially fluorinated polymer (FP) films were deposited using gas phase methods as well. After tens years of scientific researches, recently the thin PFP and FP films found several industrial applications. They include protective and antifriction coatings in traditional industries and in novel devices like organic light emitting diodes (OLED), organic field effect transistors (OFET), sensors etc.

The aim of the paper is to review in brief protective applications of vacuum-deposited fluoropolymer thin coatings.

\section{PFP coatings on metals}

PFP films were deposited on NiTi alloy surface by using the radio frequency (RF) $13.56 \mathrm{MHz} \mathrm{MSp}$ and a PTFE target [1]. The deposition parameters of PFP films including the RF power, operation gas pressure and Ar flow rate were studied. The Fourier transform infrared spectra (FTIR) and X-ray photoelectron spectra (XPS) revealed that it was feasible to prepare PFP films on NiTi alloy substrate by RF-MSp of PTFE. The FTIR and XPS results showed the presence of $\mathrm{C}-\mathrm{C},-\mathrm{CF}$, $-(\mathrm{CF}-\mathrm{CF})_{\mathrm{n}}-,-\mathrm{CF}_{2},-\mathrm{CF}_{3}$ groups etc. in the PFP films. 
The chemical structure of the PFP films changed with the deposition power and Ar flux. The films possessed the cross-linked carbon network and exhibited relatively smooth surface morphology. The surface roughness of the PFP film was $7.4 \mathrm{~nm}$. The hardness and modulus of the films were increased with the indent depth, and the surface of PFP film appeared pile-up phenomenon produced by nanoindentation test. The results showed the method to achieve low friction coefficient surface and to improve the corrosion resistance of NiTi alloy $[1,2]$. Engineering on a nano-scale was undertaken to mimic a biomaterial by forming an intercalated nanocomposite structure by sputtering of polymer with NiTi shape memory alloy [3]. PTFE was selected due to its elastic properties, low surface energy and low interactions with water, stability and chemical resistance. NiTi shape memory alloy allows the coatings to be energy absorbent and thus suitable in load bearing situations. The coatings are aimed to withstand variable biological environments whilst maintaining their characteristics. The nano-intercalated structures are characterized by their wettability, friction coefficients, chemical composition, and morphology. Intercalation of polymer with energy-absorbing alloys uncovered a set of material systems that offered characteristics such as selfhealing of hierarchal tissue in the body. The reformation of PTFE following MSp was confirmed by FTIR spectra. PTFE showed a surface interaction with NiTi, thus formed stable coating. Surface interactions are evident by the hydrophobic behaviour of films, since the water contact angle was close to $86^{\circ}$, which lies inbetween those of PTFE and NiTi. The nanocomposite films have a measured friction coefficient below 0.2 which does not vary with the layer thickness [3]. Pd capped $\mathrm{Mg}-\mathrm{Ni}$ thin films were prepared on glass substrates by DC-MSp for a switchable mirror. In order to improve the switching durability, PTFE film was deposited on it by RF-MSp. Sputtering of PTFE was carried out at the power $30 \mathrm{~W}$ in the $\mathrm{Ar}$ and $\mathrm{CF}_{4}$ mixed gas discharge. PTFE film with $900 \mathrm{~nm}$ thickness on glass substrate had transmittance of $90 \%$. PTFE film showed the excellent protection against the oxidization of $\mathrm{Mg}$ and gave the 1000 switching cycles by 4 vol.\% hydrogen [4]. PTFE coatings were deposited using MSp on sand blasted glass substrates, which had roughness within the range 50 to $7000 \mathrm{~nm}$. The PTFE coatings with $R_{a}=4500 \ldots 4800 \mathrm{~nm}$ were found to be superhydrophobic with the static contact angle close to $152^{\circ}$ and low contact angle hysteresis $\left(2^{\circ}\right)$. The superhydrophobicity was attributed to the presence of dual scale roughness, densely packed microstructure and the presence of $\mathrm{CF}_{3}$ groups [5]. Superhydrophobic properties were achieved for RF-MSp PTFE coatings deposited on etched aluminum (Al) surfaces. Surfaces with water contact angles higher than $150^{\circ}$ were achieved on PTFE films of less than 10-nm thickness, coating the $\mathrm{Al}$ surfaces, etched for ten minutes with hydrochloric acid ( $\mathrm{HCl}, 7$ wt.\% in $\mathrm{H}_{2} \mathrm{O}$ ). Authors concluded that the presence of patterned morphology, along with the low surface energy on PTFE coating, made the Al surface highly superhydrophobic [6]. A hydrophobic property was introduced to vertically aligned hydrophilic platinum $(\mathrm{Pt})$ nanorods. The nanorod arrays were deposited on flat silicon substrates using a sputter glancing angle deposition technique (GLAD). Then, a thin layer of PTFE was partially deposited on the tips of Pt nanorods by RF-MSp of PTFE at the glancing angle $\theta_{\text {dep }}=85^{\circ}$ for different deposition times. PTFE also was deposited on Pt nanorods at normal incidence $\left(\theta_{\text {dep }}=0^{\circ}\right)$ for comparison. Morphological and elemental analyses of $\mathrm{Pt} /$ Teflon nanocomposites were carried out using scanning electron microscopy (SEM) and energy dispersive X-ray analysis. It was found that the GLAD technique is capable of depositing ultrathin isolated PTFE structures on selective regions of nanorod arrays due to the shadowing effect during obliquely incident deposition. Pt nanorods with PTFE nanopatches exhibited contact angle values as high as $138^{\circ}$, indicating a significant increase in the hydrophobicity of originally hydrophilic Pt nanorods that had an angle of about $52^{\circ}$. The enhanced hydrophobicity of the nanorod/PTFE composite is attributed to the presence of PTFE coating, which imparted a low surface energy. Surface energy calculations were performed on $\mathrm{Pt}$ nanorods, PTFE thin film, and Pt/PTFE composite by using the two-liquid method to confirm the contact angle measurements. Furthermore, a new contact angle model utilizing Cassie and Baxter theory for heterogeneous surfaces was developed in order to explain the enhanced hydrophobicity of Pt/PTFE nanorods. According to the model, the solid-liquid interface is mainly at the PTFE tips, when the composite nanorods are in contact with water [7, 8]. Similar results were obtained on hydrophobicity of the PTFE coated $\mathrm{ZnO}$ nanorods by using RF-MSp of PTFE [9]. Authors showed that UV irradiation led to increase of wettability of PTFE-coated $\mathrm{ZnO}$ nanorods.

\section{FP coatings applications in bio/medicine}

Different methods for deposition of the thin polymer films from gas phase (including HW CVD) for various applications were reviewed in [10]. The paper contains data concerned PFP, FP and other industrially valuable polymer films applications in the fields of sensors, medicine, biotechnology, microelectronics, organic electronics, microfluidics etc. A vapor phase deposition system was designed to deposit uniform, conformal and thin films of FPs (Cytop and Fluorinert) and fluorosilane (PFPTES) inside silicon nanochannels [11]. Vapor phase deposition has advantages over liquid phase deposition, since vapor permeates more efficiently into nanochannels. The films are desirable to control the hydrophobicity of the surface and to prevent undesired protein adsorption or cell interactions. The films were characterized by means of a contact angle analyzer for hydrophobicity and an ellipsometer for film thickness. Atomic force microscopy (AFM) was used to study the 
surface, adhesive and frictional properties of these films. The surface properties of the films on silicon were governed by the deposition temperature and annealing time, but there is a little effect due to the chamber pressure. The increase in the static contact angle was observed for Cytop, Fluorinert and PFPTES films as compared to that in silicon. The static contact angles of the Fluorinert films were higher than those of Cytop films. While the Cytop and Fluorinert were deposited as a multilayer film on silicon, PFPTES was deposited as a monolayer with the silane reaction controlling the processing conditions. In the nanochannel system, coating on a monolayer level is favored due to the nanochannel dimensions. The roughness values and the standard deviation of the values of Cytop films on silicon were decreased as a function of temperature. Reduction in coefficient of friction and in adhesive force was found for all three film materials as compared to the silicon substrate [11].

The pulsed laser-assisted surface modification (PLASM) method for producing biocompatible films was developed at Nanotherapeutics [12]. Using PLASM, a 5 to $10-\mu \mathrm{m}$ thick PTFE coating was applied to glass slides in 10 minutes. Coated and uncoated slides were then seeded with DH5a E. coli to test for reduction of bacterial adhesion. Compared to uncoated slides, the PTFE-coated glass slides demonstrated reduced adhesion onto PTFE after one hour of bacteria incubation.

A multistep process was developed in order to isolate the stainless steel surface from the body fluid by depositing a thin $(\sim 35 \mathrm{~nm})$ PP PFP film. Deposition of thin films on outer and inner surfaces of millimetric sized diameter stainless steel tubes by RF pulsed PP was made for coronary stents. A mixture of hexafluorethylene $\left(\mathrm{C}_{2} \mathrm{~F}_{6}\right)$ and hydrogen in a post-discharge was used. At floating potential, a PFP coating was deposited, varying in chemical composition and thickness along the tube axis. The application of $-400 \mathrm{~V}$ bias voltage led to outer surfaces with a PFP coating uniform in thickness and homogeneous in composition, along the full length of the tube $(10 \mathrm{~mm})$. Despite application of a negative bias, the covering efficiency for the inner surface was lower as compared with the outer surface, with PFP films homogeneous in both composition and thickness until half length of the tube but thickness decreasing afterwards [13]. Produced PFP films resisted to plastic deformations up to $25 \%$. In order to abide to strict FDA regulations, the film must be stable after implantation. The compositional and morphological changes of the PFP films were examined during ageing in a pseudophysiological medium, a phosphate buffer solution, by angle-resolved XPS, FTIR and AFM. The evolution of the ageing process is described as follows: defluorination and crosslinking yielded an oxidized protective top layer onto the films, which shows further degradation [14]. The effect of $25 \%$ plastic deformation on stability and ageing characteristics of the PFP coating were also verified. The film partially lost its hydrophobicity in the initial phase of the incubation process, which enabled buffer to penetrate throughout the film. Results proved that flat specimens to be covered with a metastable protective outermost layer, which initially inhibited degradation, but as a function of time started to dissolve into the ageing medium. Due to the deformation procedure, surface chemistry has undergone compositional changes, which accelerates the ageing process. Degradation and oxidation of the PFP films were observed. However, no metallic elements were detected by XPS, indicating that the film was protected and oxidation of underlying stainless steel was delayed. The presence of nano-pinholes induced water infiltration and, therefore, film degradation. To minimize the impact of these nano-defects, a multi-layer should be formed by modifying the plasma polymerization parameters during the deposition process. PP PFP coatings were described in the short review with reference to their applications for biomaterials and biomedical devices [15]. PE CVD processes coupled with micro/nano patterning and texturing procedures were described, as well as the effect of surface chemical composition and texture on general behavior of cells in vitro. PFP coatings were deposited at the inside walls of Dacron vascular grafts and on other conventional polymers, and positive results were obtained on their non-thrombogenic character, resistance to platelet adhesion and activation, blood compatibility and adsorption-retention properties toward blood proteins. Technical approaches for the modification of vascular stents and neural implants with PFP films were described in [16]. Optimization of RF-MSp parameters allowed modification of the surface morphology and chemical functionality in order to achieve the desired performance. Thin composite films were co-deposited from $316 \mathrm{~L}$ stainless steel and PTFE targets. The functionally graded thin nanocomposite film, consisting of a metallic composition near the stent and a PFP material in the outmost surface, was produced. These films allowed fast re-endothelisation of the coated stent when compared to the uncoated one. The modification of surfaces with PTFE/Au thin films intended to address two distinctive problems. The first one related to ensuring the correct positioning of some implantable devices into the human body. The solution is to introduce in the coating, nanomaterials with a high electron density like to that in gold. The other problem is rejection of the "foreign" materials by the human body. This process begins with adsorption of biological proteins that, due to the subsequent denaturation, triggers rejection biological pathways. The use of a PFP material is more favorable in preventing the loss of protein $3 \mathrm{D}$ conformation. Co-deposition of these two materials produced a nanocomposite structure. Its surface permitted adsorption of bovine serum albumin without protein denaturation. Another research aim was to develop an antimicrobial coating. The thin film with organic/inorganic matrix doped with antimicrobial metal is being studied. Co-deposition of hydroxyapatite and 
PTFE, doped with silver, which creates surfaces with inhomogeneous phase distribution could be cited as an example. This graded film structure appeared to induce on the same surface the ability to simultaneously promote and inhibit the growth of selected bacterial strains [16]. Several industrial enterprises are depositing the PFP films on medical devices from gas phase. Applications of the PFP films are the antifriction, bioinert and stable protective coatings on medical instruments and devices: stents from stainless steel, needles, nozzles, orifices, sensors, guide-wires. Surface Engineering Associates apply PFP by PP onto the above mentioned surfaces using ultra-high vacuum processing performed at room temperature [17]. Thierry Corporation deposits PFP films with PP on implants, contact lenses, hearing aids, cell culturing etc. [18].

\section{Surface passivation by PFP coatings}

PFP films of about 4-nm thickness were deposited on the HCI-etched (100)-oriented GaAs monocrystalline substrates via RF sputtering of PTFE target. XPS and time-of-flight secondary mass spectroscopy indicated that the PTFE film was oxygen-free and consisted of $-\left(\mathrm{CF}_{2}\right)_{\mathrm{n}}-$ units with different end groups. The deposited film, having a dielectric constant comparable to that of the bulk PTFE (K 2.1), could passivate the HCIetched GaAs (100) substrate. The growth of the oxide layer was effectively hindered by the PTFE barrier, when the HCI-etched GaAs (100) surface was exposed to air for several hundred hours. The surface oxidation rate was reduced significantly in the presence of the PTFE barrier when the HCI-etched GaAs (100) was exposed to water and the $\mathrm{H}_{2} \mathrm{O}_{2}$ solution. The $180^{\circ}$ peel adhesion test showed that the PTFE film adhered strongly to the GaAs (100) surface [19]. The like results were obtained later but on (100) Si substrate by RF sputtering [20], by LA and pulsed electron beam deposition [21].

Process for the production of hydrophobic layers on optical substrates was described in the patent [22]. The optical substrates that have an alkaline earth metal fluoride or alkali metal fluoride layer as the outermost layer or consist of alkaline earth metal fluorides or alkali metal fluorides were covered with the PFP film by thermal vapor deposition with polyfluorohydrocarbons in high vacuum with preference to PTFE. The mixtures from PFP, oxide and metal powders were made, then used were the composite granules for heating in vacuum to deposit PFP films. But for the PTFE, the inorganic matrix is not necessary, for thermoplastic (fluoro)polymers the same method was described before in [23]. The use of FP film to protect $\mathrm{Si}$ regions from anodization in HF electrolyte was investigated. The film was studied for two different substrates $\left(p^{+}-\right.$and $n$-type $\mathrm{Si})$ under anodic bias: no porous $\mathrm{Si}$ was observed under the mask (except for the border over-etching that cannot be avoided). This mask presented many advantages compared to others, especially its fast and non-altering removal after electrochemical etching. This type of masking layer can be employed for microelectronic applications such as MEMS, Si/PolySi hybrid substrates etc. Due to its chemical resistance, FP may be employed as a protective layer for the underlying ones in the backend electrochemical etching process [24].

\section{PFP coatings on fabrics}

The PTFE film was deposited by RF-MSp on silk fabric to improve its hydrophobicity. The wettability of the fabric was characterized through measuring the surface contact angle by a dynamic sessile analysis technique. The contact angle of the PTFE coated fabric showed an increase from $68^{\circ}$ to about $138^{\circ}$. The larger sputtering pressures brought less contact angle hysteresis [25]. The different films exhibited contact angles from $119^{\circ}$ up to $153^{\circ}$, depending on the preparation conditions. To obtain superhydrophobic surface, a rough PTFE film with structure gradient was deposited through controlling the operation pressure. The increase of surface roughness with the operation pressure was achieved. The presence of dual size PTFE clusters on the surface of the substrate enhanced superhydrophobicity [26].

\section{PFP coatings on carbon nanotube (CNT) “films”}

The superhydrophobic surface was made using the nanoscale roughness inherent to a vertically aligned carbon nanotube forest, in which the surface of the CNTs covered with thin PTFE film by HW CVD. Superhydrophobicity was achieved down to the microscopic level where spherical, micrometer-sized water droplets were suspended on top of the CNT forest [27]. The PTFE coating was deposited onto the forest of CNT with a HW CVD method. The height of carbon nanotubes was coated with a thin PTFE film. Using an array of stainless steel filaments resistively heated to $500{ }^{\circ} \mathrm{C}$, hexafluoropropylene oxide gas was decomposed to difluorocarbene $\left(\mathrm{CF}_{2}\right)$ biradicals. These radicals polymerized into PTFE on the nanotube surfaces that were kept at room temperature. Another source of the difluorocarbene biradicals was the PTFE bulk heated in vacuum [28]. CNT forest also was coated by RF-MSp of PTFE [29]. These films can be used in batteries, aircrafts, artificial muscles.

\section{PFP films in sensors}

Element One's hydrogen indicating pigments are the coatings for the detection of hydrogen, which change color, either reversibly or non-reversibly, to give both current and historical information about leaked hydrogen. An optical thin film indicator consists of a transparent polymer substrate, a 500-nm thick layer of nano-porous tungsten oxide, 3-nm layer of palladium catalyst, and a 100-nm thick film of PTFE. The PTFE film partially protects the catalyst from poisoning by atmospheric pollutants. PTFE top layer in the indicator 
helps to retard this contamination, but does so imperfectly. Thicker and more dense protective film slows the rate of contamination more, but also slows the indicator response. All of the layers are deposited by vacuum deposition [30]. The similar type of sensor with PTFE protective film was developed in India [31] and in Japan [32], but they are used for electrical detection of changes occurred in the multilayered film in hydrogen presence. The sensors made in Japan use a multilayered structure: in the case of PI-10/PTFE-10/Pd-Pt/T-TiO sensor, the additional coating of PI-10 on the PTFE increased the magnitude of wet air/dry air sensor response at temperatures lower than $200^{\circ} \mathrm{C}$, in comparison with that of $\mathrm{Pd}-\mathrm{Pt} / \mathrm{T}-\mathrm{TiO}_{2}$, leading to the enhancement of the interference from humidity on the $\mathrm{H}_{2}$ response. The similar hydrogen sensors are designed in Netherland [33-35]. They are investigating why addition of small amounts of $\mathrm{Zr}$ to an $\mathrm{Y}$ thin film results in a significant change in its hydrogen sorption properties, and deposition of MSp PTFE on Pd-based thin film catalysts lead to a profound increase in the hydrogenation and dehydrogenation kinetics.

ADZ Nagano deposits PTFE layers with the thickness between 0.5 to $1 \mu \mathrm{m}$, which has no adverse performance influence on stainless steel membrane and thin film semiconductor diaphragm [36]. The PTFE film improves the contamination resistance of the IP68 rated sensor and significantly extends the cleaning intervals required for maintenance. A row of equipment for coatings deposition by plasma polymerization, including PFP ones, are made by Diener electronic GmbH [37].

\section{PFP protective coatings for OFET and OLED}

The pentacene layer in an OFET was encapsulated with $1.5-\mu \mathrm{m}$ thick film of PTFE and stored for 4 months in laboratory conditions. The on-current value was reduced from an initial value of $-6.2 \mathrm{~mA}$ to $-1.1 \mathrm{~mA}$ after 4 months. Compared to unprotected samples, the rate of degradation was strongly reduced. A positive threshold voltage shift occurred, indicating that humidity can be excluded as a degradation factor. Oxygen may have diffused through the PTFE layer [38,39]. The PFP are used in organic-inorganic multilayered encapsulations for OLEDs developed by GVD, Selvac etc. [40]. Several advantages of the PFP use instead of Parylene were mentioned. The finishing for the printing circuits boards industry is based on the use of a thin PFP film as a protective coating to preserve solderability of the circuit board between manufacture and assembly. The film extends the shelf life of the printed circuit board by preventing oxidation and corrosion, and ensures excellent solderability during assembly. The PFP film is applied using PP. The film was applied directly onto copper, or can be used in conjunction with other surface finishes to act as a corrosion inhibitor [41]. The invention [42] described the electro-optical devices, including organic electroluminescent devices that are protected from adverse environmental conditions with FP. The term "fluoropolymer" referred to the class of paraffin polymers that have some or all of the hydrogen replaced by fluorine. Among FP that are adaptable for use within the present invention, there are PTFE, fluorinated ethylene etc. Apple just put into use the PFP coating produced by PP for its smartphone waterproofing [43].

\section{PTFE matrix protects dye molecules inside it}

Since the end of the $20^{\text {th }}$ century, PTFE thin films, produced using thermal decomposition of the bulk PTFE in vacuum with activation of the vapours by accelerated electrons [44] were used in optical recording media as a sublayer [45]. The metal-filled thin PTFE films also were obtained and tested for optical memory with a long lifetime [46]. Farther, this method was applied for production of dye-filled PTFE films [47-56]. At first, the well known and stable phthalocyanine (Pc) compounds were introduced into the PTFE matrix. The PTFE matrix kept the dye in the molecular state at small dye concentrations (Fig. 1a). The stability of the composite against aggregation during heating was found out (Fig. 1a). The PTFE matrix kept VO-Pc molecules from transition from low to high temperature phase (Fig. 2). Later the different evaporable organic compounds were used: squaraine (Fig. 1b), acidochromic dyes of stillbene and polymethine kinds, photochromic spirocompounds and various polymethine compounds [47-56].
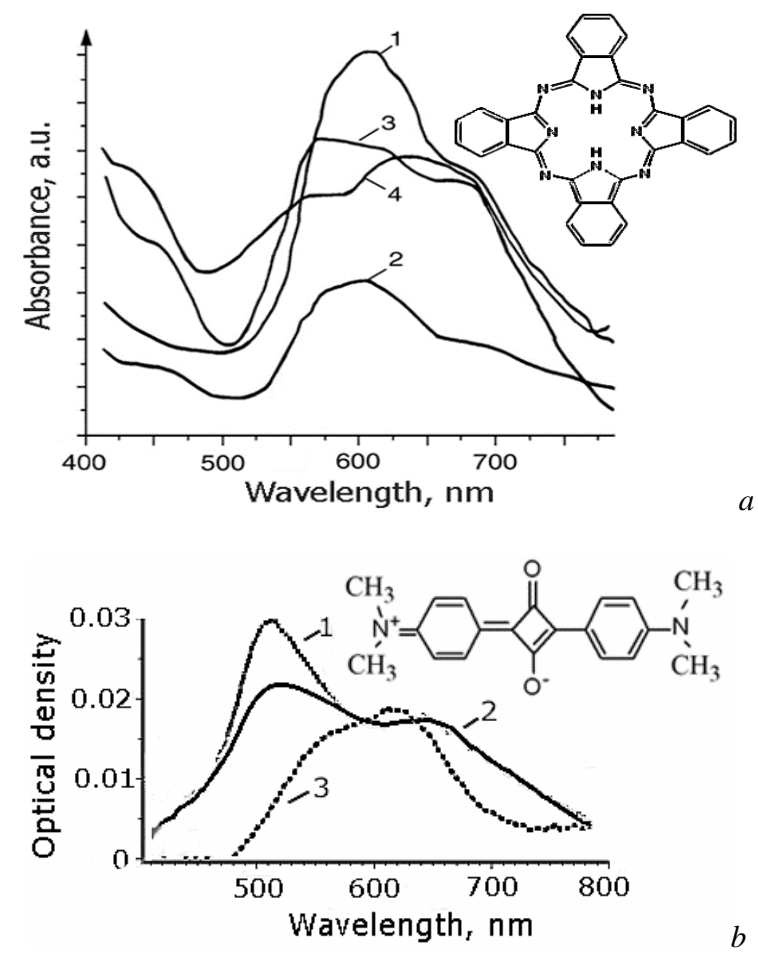

Fig. 1. a) Optical spectra of $\mathrm{H}_{2} \mathrm{Pc}$-in-PTFE films after annealing at $200{ }^{\circ} \mathrm{C}$ for 4 hours in air. 1-4 depicts dye concentration decrease. Spectra 3 and 4 were scaled for clarity. b) Spectra of squaraine molecules: 1 - on PTFE sublayer, 2 on glass, 3 - in PTFE matrix. 

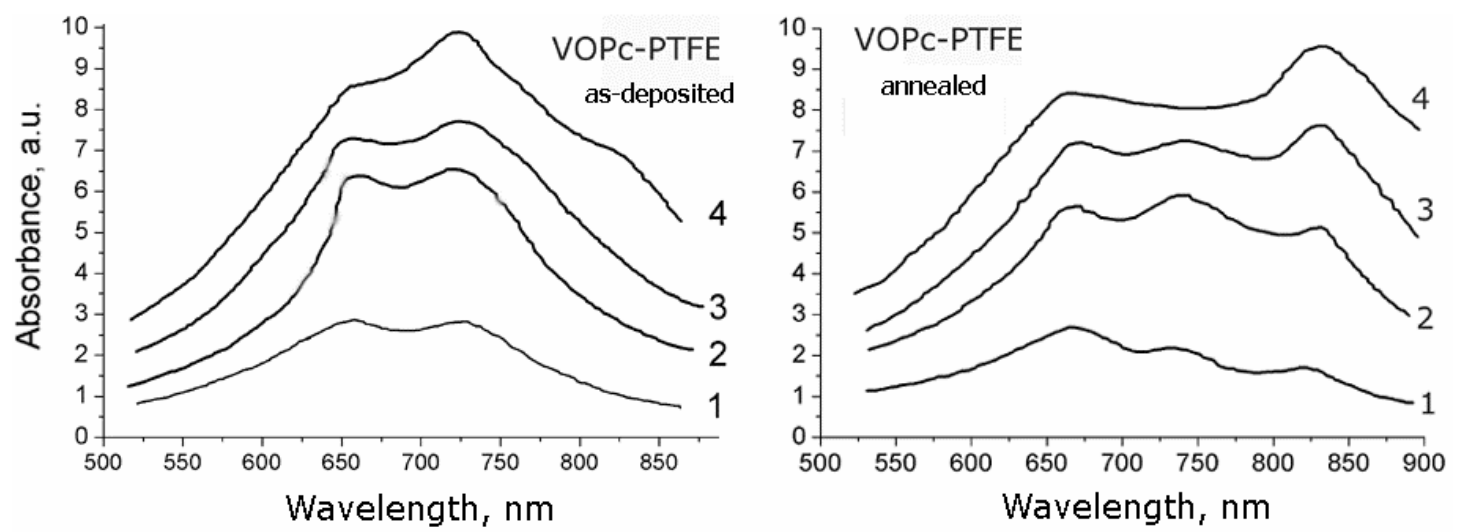

Fig. 2. Spectra of VOPc in PTFE matrix: 1-4 shows VOPc concentration increase.

Heating in air the dye-filled PTFE thin films revealed their extreme stability. Spectra evolution during heating in air of various dye-filled polymer films with dye's concentration within the range $10 \ldots 15$ vol.\% were presented in [51]. The effect of the polymer matrix on the film optical spectra changes during heating was clearly seen. The dye molecules aggregation in polyparaphenylene sulphide (PPS) matrix was significantly stronger, than in PTFE matrix. At the temperature, at which dye-filled PPS films became transparent, dye-filled PTFE films still revealed some optical absorption. The film with PTFE matrix was not transparent up to $260^{\circ} \mathrm{C}$ (Fig. 3). The appearance of the band in the blue region can be explained by decomposition of the dye molecules. The decomposition products were kept in the PTFE matrix.

PTFE films filled with spirocompounds were produced by co-deposition in vacuum. The spectra of the as-deposited and UV-irradiated films are shown in Fig. 4. Transmission spectra of the PTFE films filled with spyropiran molecules showed that photochromic transformation rate was enhanced in proportion to the dye concentration decrease [52]. But if the dye concentration was less than $10 \%$ vol., no transformation was detected at all. The spyropiran-filled PTFE films exhibited high photo- and thermal stability. Kinetics of spontaneous spiropyran bleaching and photodegradation are presented in Fig. 5. Spontaneous transformation of the photoinduced merocyanine form into the initial spiropyran form was absent. The photochromic dyePTFE films were characterized with high stability to the irreversible photochemical transformations. At the same conditions of irradiation, photodegradation of spiropyran-filled PTFE film with the thickness close to $100 \mathrm{~nm}$ was substantially smaller as compared with that in the spiropyran-filled PMMA films [52, 53].

The PTFE films filled with acidochromic dyes were immersed in the different aggressive vapors [53]. All types of dyes in the PTFE matrix showed no any light absorption or luminescence changes in aggressive vapors. The same all dyes but in PPS matrix revealed changes in absorption and luminescence spectra under the same conditions. It was found that the PTFE films

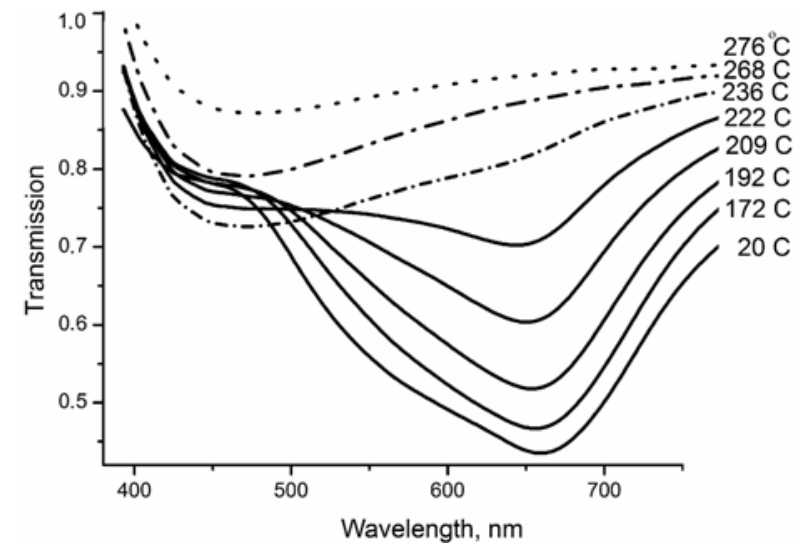

Fig. 3. Optical spectra evolution during heating in air of squaraine-filled thin PTFE film.

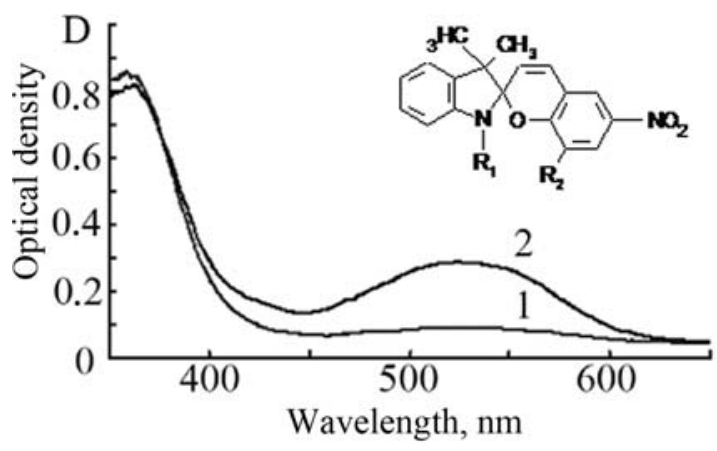

Fig. 4. Optical absorption spectra of the spirocompound in the PTFE matrix $\left(\mathrm{R}_{1}=\mathrm{CH}_{3} ; \mathrm{R}_{2}=\mathrm{OCH}_{3}\right)$ before (1) and after (2) UV irradiation. 
filled both with inorganic and dye nanoparticles (NPs) were sensitive toward aggressive vapors. Film with the PTFE matrix filled with Au NPs and 1-2,5-(-4dimethylaminostyryl)-pyrazine molecules revealed changes in absorption spectrum, luminescence was suppressed. Therefore, the PTFE in thin film form protects the dye molecules incorporated in it toward all environmental factors. It is hardly possible to explain the
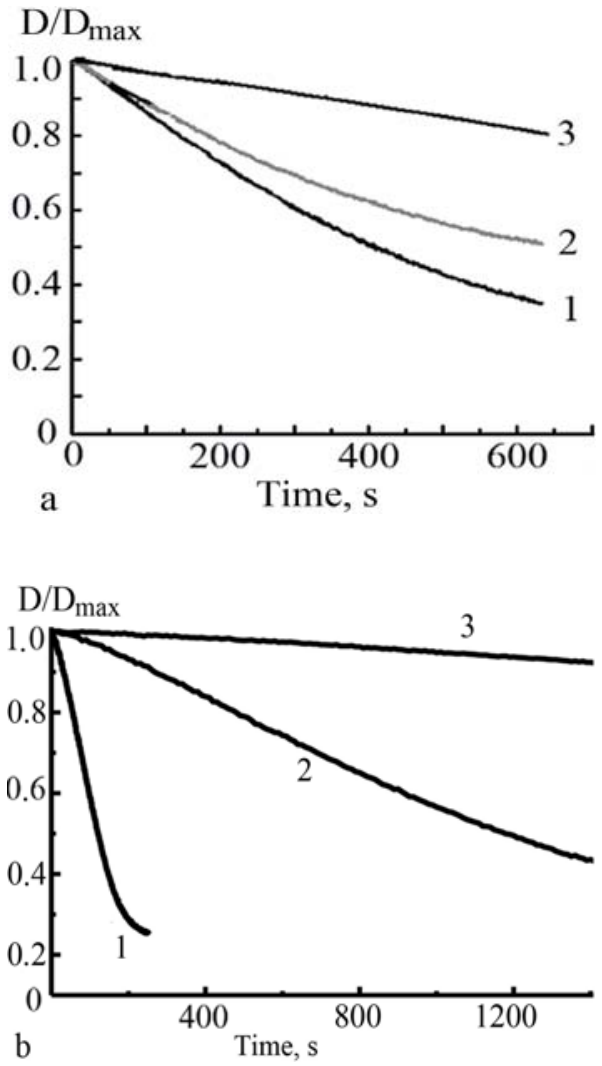

Fig. 5. Kinetics of spontaneous bleaching (a) and photodegradation (b) of film of the spiropyran in PMMA matrix at $25^{\circ} \mathrm{C}$, prepared by spin coating: $1-C=0.1$ weight $\%$, $\Delta D^{\text {phot }}=0.6 ; 2-C=25$ weight $\%, \Delta D^{\text {phot }}=0.23 ; 3-$ in PTFE matrix $\left(C=10\right.$ weight $\left.\%, \Delta D^{\text {phot }}=0.6\right)$.
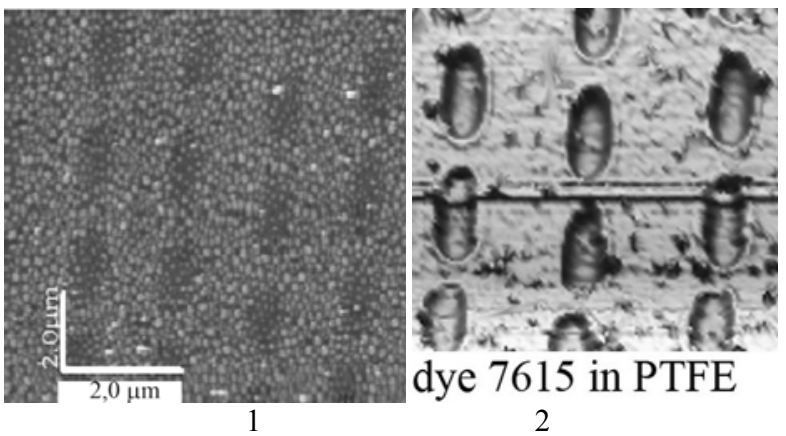

Fig. 6. 1 - pure dye film with dye molecules aggregation after laser irradiation; 2 - dye in PTFE film with no aggregation around the holes. results as the sum of the dye and PTFE properties. The diffusion of vapors and gases should take a few second through film with the thickness of several tens of nanometers. The inorganic NPs can absorb gaseous molecules, in this way supplying them into the PTFE film toward dye molecules. The PTFE macromolecules in the deposited film contained electric charge. Perhaps, this charge did not allow molecules of the gasses penetrate into film bulk. But what is the mechanism for suppression of phototransformations of the spirocompounds in charged PTFE environment is not clear.

The dye-filled PTFE thin film was tested for thermal lithography by focused laser beam with $405 \mathrm{~nm}$ wavelength. The round shaped holes were obtained with their diameter far smaller (down to $100 \mathrm{~nm}$ ) than the diameter of laser beam, due to high melting temperature and high viscosity of the PTFE melt. The PTFE matrix preserved the dye molecules in the nearby hole heated zone from aggregation (Fig. 6). The film is useful for superhigh density optical memory [57-59].

The new properties should be expected for films of the metal and dye NPs in the PTFE matrix, which should summarize both dye and metal NPs properties. The codeposition of dye, metal and PTFE from three sources resulted in formation of both dye and metal NPs in PTFE matrix with weak interaction between metal NPs and dyes molecules (Fig. 7) [59].

The increase of the initial transmission of the glass substrate with composite film growth in the red region was due to antireflective action of the PTFE film. In this region the NPs own absorption was smaller than PTFE antireflective action. We suggested that the use of organic compounds, which are able to interact with metal NPs surface, can lead to the films with new properties. For Au NPs the use of the sulphur-terminated compounds leads to compound-NPs interaction (Fig. 8). Such films were tested as sensors for aggressive gases and showed promising properties [50].

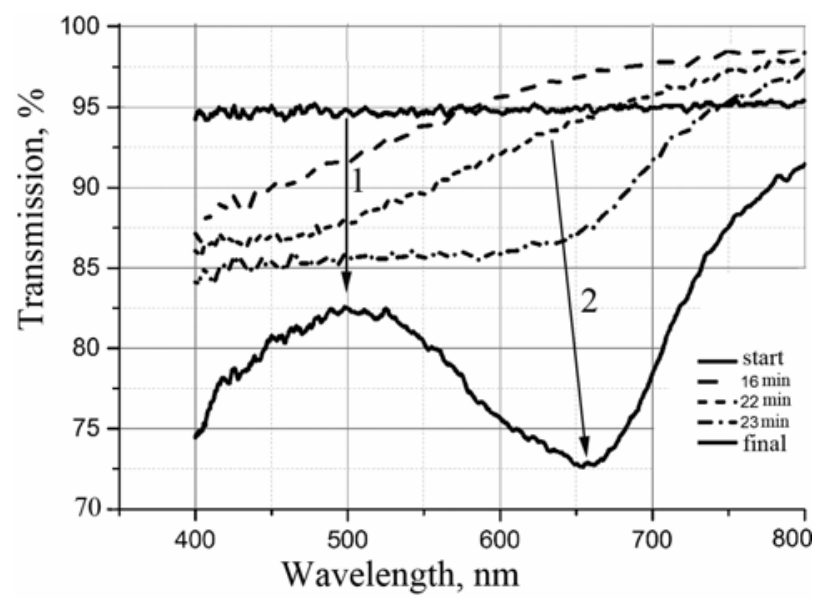

Fig. 7. Optical spectra of the PTFE film filled with squaraine molecules and Au NPs, recorded in situ during film growth: 1 - Au NPs plasmon band maximum; 2 - absorption maximum of squaraine molecules (Fig. 1b). 


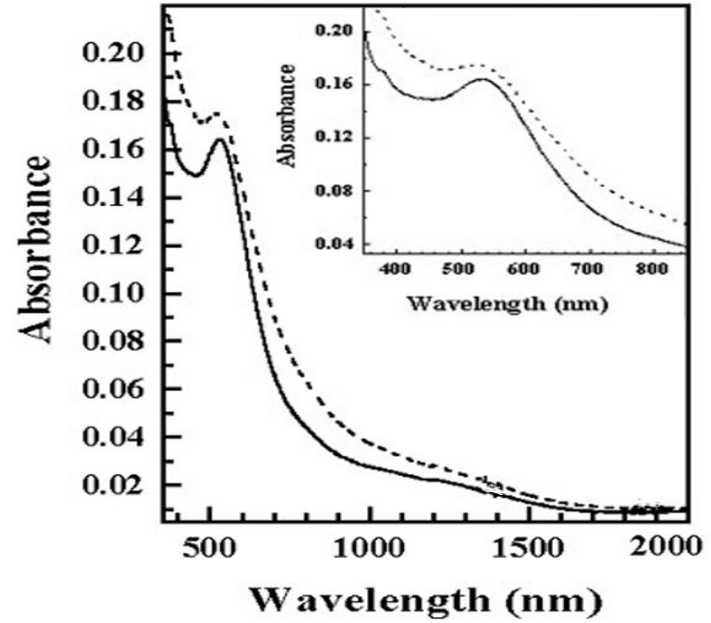

Fig. 8. Comparison of absorption spectra between the asdeposited film (continuous line) and after its functionalization with dodecanethiol (dashed line).
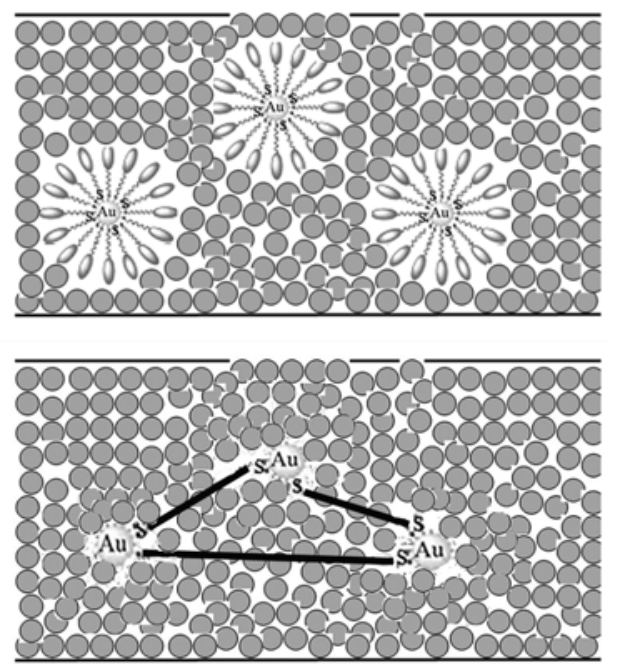

Fig. 9. Possible organized structures of dye and metal NPs in the PTFE matrix: 1 - Au NPs with dye "fur"; 2 - Au NPS related with dye nanowires.

It was suggested, that if the specially designed organic molecules will be co-deposited with metal, the organized structures (Fig. 9) with new properties can be grown in the PTFE matrix [60]. It is expected the high stability of these structures provided by the PTFE matrix.

\section{Conclusions}

The thin perfluoropolymer films deposited from the gas phase are used for encapsulation and protection of the surface of various materials in different novel devices. Organic molecules in the PTFE matrix represent new class of materials that reveal high stability and can be considered as promising for various optical applications.
References

1. L. Li, F.T. Zi, Polymer thin films, prepared by RF sputtering of PTFE on NiTi alloy // Mater. Sci. forum, 561, p. 1229-1232 (2007).

2. L. Li, F.T. Zi, Y.F. Zheng, The characterization of fluorocarbon films on NiTi alloy by magnetron sputtering // Appl. Surf. Sci. 255, p.432-434 (2008).

3. S.S. Anjum, J. Rao and J.R. Nicholls, Polymer (PTFE) and shape memory alloy (NiTi) intercalated nano-biocomposites // Intern. Conf. on Structural Nano Composites (NANOSTRUC 2012), IOP Conf. Series: Mater. Sci. and Eng. 40, p.1-7 (2012).

4. S. Bao, K. Tajima, Y. Yamada, M. Okada and K. Yoshimura, Polytetrafluoroethylene (PTFE) topcovered Mg-Ni switchable mirror thin films // Materials Trans. 49(8), p. 1919-1921 (2008).

5. N. Selvakumar, H.C. Barshilia, M. Ramesh, K.S. Rajam, Effect of substrate roughness on contact angle of sputter deposited superhydrophobic PTFE coatings // Conference Surface Modification Technologies XXIII, Ed. T.S. Sudarshan, U.K. Mudali and B. Raj, India, 2009, p. 1-7.

6. Hwa-Min Kim, J. Jang and S. Sohn, Superhydrophobicity of PTFE films coated on an etched Al surface by using a RF-magnetron sputtering method // J. Korean Phys. Soc. 57(5), p. 1281-1284 (2010).

7. W.J. Khudhayer, R. Sharma and T. Karabacak, Hydrophobic metallic nanorods with Teflon nanopatches // Nanotechnology, 20, p. 1-9 (2009).

8. W.J. Khudhayer, R. Sharma and T. Karabacak, Hydrophobic metallic nanorods coated with Teflon nanopatches by glancing angle deposition // Mater. Res. Soc. Symp. Proc. 1188, 1188-LL09-03 (2009).

9. H. Zheng, M. Breedon, K. Kalantar-zadeh, $\mathrm{UV}$-induced wettability change of Teflon-modified $\mathrm{ZnO}$ nanorod arrays on $\mathrm{LiNbO}_{3}$ substrate // ICONN, IEEE, p. 218-2212 (2008).

10. G. Ozaydin-Ince, A.M. Coclite and K.K. Gleason, CVD of polymeric thin films: applications in sensors, biotechnology, microelectronics/organic electronics, microfluidics, MEMS, composites and membranes // Rep. Prog. Phys. 75, p. 1-41 (2012).

11. K.K. Lee, B. Bhushan, D. Hansford, Nanotribological characterization of fluoropolymer thin films for biomedical micro/nanoelectromechanical system applications // J. Vac. Sci. Technol. A, 234, p. 804-810 (2005).

12. J.D. Talton, B. Eppler, and J.M. Fitz-Gerald, Pulsed laser-assisted surface modification / functionalization // Surfaces in Biomaterials, 8(2), p. 4 (2003).

13. V. Nelea, S. Holvoet, S. Turgeon and D. Mantovani, Deposition of fluorocarbon thin films on outer and inner surfaces of stainless steel mini-tubes by pulsed plasma polymerization for stents // J. Phys. D: Appl. Phys. 42, p. 1-9 (2009). 
14. S. Holvoet, P. Chevallier, S. Turgeon and D. Mantovani, Toward high-performance coatings for biomedical devices: Study on plasma-deposited fluorocarbon films and ageing in PBS // Materials, 3, p. 1515-1532 (2010).

15. P. Favia, Plasma deposited coatings for biomedical materials and devices: Fluorocarbon and PEO-like coatings // Surface \& Coatings Technol. 211, p. 5056 (2012).

16. A.P. Piedade, J. Nunes, M.T. Vieira, Thin films with chemically graded functionality based on fluorine polymers and stainless steel // Acta Biomaterialia, 4, p. 1073-1080 (2008).

17. http://www.seac1.com/

18. http://www.thierry-corp.com/pecvd-plasma-coating

19. Yan Zhang, E.T. Kang, K.G. Neoh, S.S. Ang, and A.C.H. Huan, Surface passivation of (100)-oriented GaAs with ultrathin fluoropolymer films deposited by radio frequency magnetron sputtering of poly(tetrafluoroethylene) // J. Electrochem. Soc. 150(3), p. 53-59 (2003).

20. D.S. Bodasa, A.B. Mandalea, S.A. Gangala, Deposition of PTFE thin films by RF plasma sputtering on $\langle 100\rangle$ silicon substrates // Appl. Surf. Sci. 245(1-4), p. 202-207 (2005).

21. J. Piwowarczyk, R. Jędrzejewski, K. Kwiatkowski, J. Baranowska, PTFE thin films obtained by pulsed electron beam deposition and pulsed laser deposition methods // 9-th Symposium on Vacuum Based Sci. and Technol. November, 17-19, 2015, Koszalin-Kołobrzeg, Poland, p. O14.

22. U. Anthes, R. Dpmbrowski, M. Fritz, Method and agent for producing hydrophobic layers on fluoride layers // Patent US 6,783,704, B1, Aug. 31, 2004.

23. K.P. Gritsenko, V.S. Gritsenko, Evaporation elements for polymer coatings deposition by vacuum evaporation // Powder metallurgy, 2, p. 98100 (1990), in Russian.

24. T. Defforg, M. Capelle, F. Tran-Van and G. Gautier, Plasma-deposited fluoropolymer film mask for local porous silicon formation // Nanoscale Res. Lett. 7(1), p. 344-351 (2012).

25. F. Huang, Q. Wei, Ya Liu, W. Gao, Y. Huang, Surface functionalization of silk fabric by PTFE sputter coating // J. Mater. Sci. p. 8025-8028 (2007).

26. F.L. Huang, Q.F. Wei, W.Z. Xu and Q. Li, Preparation and characterizations of PTFE gradient nanostructure on silk fabric // Surface Rev. and Lett. 14(4), p. 547-551 (2007).

27. G.A.J. Amaratunga, W.I. Milne, G.H. McKinley and K.K. Gleason, Superhydrophobic carbon nanotube forests // Nano Lett. 3(12), p. 1701-1705 (2003).

28. E.M. Tolstopyatov, Physical backgrounds of the dissotiative formation of the polymer coatings // Thes. Dr. Sci., Gomel, 2007 (in Russian).
29. T. Barfels, Carbon Nano-Tubes and NanoDiamonds in applied research // Cross beam workshop, Zeiss, Dez. p. 29 (2013).

30. www.elem1.com

31. S.C. Gadkari, Manmeet Kaur, V.R. Katti, V.B. Bhandarkar, K.P. Muthe, and S.K. Gupta, Solid state sensors for toxic gases // Founders day special issue, p. 49-60 (2005).

32. G. Yamamoto, T. Yamashita, K. Matsuo, T. Hyodo and Y. Shimizu, Effects of polytetrafluoroethylene or polyimide coating on $\mathrm{H}_{2}$ sensing properties of anodized $\mathrm{TiO}_{2}$ films equipped with $\mathrm{Pd}-\mathrm{Pt}$ electrodes // Sensors and Actuators B: Chemical, 183, p. 253-264 (2013).

33. R.J. Westerwaal, S. Gersen, P. Ngene, H. Darmeveil, H. Schreuders, J. Middelkoop, B. Dam, Fiber optic hydrogen sensor for a continuously monitoring of the partial hydrogen pressure in the natural gas grid // Sensors and Actuators B, 199, p. 127-132 (2014).

34. T. Mak, R.J. Westerwaal, M. Slaman, H. Schreuders, A.W. van Vugt, M. Victoria, C. Boelsma, B. Dam, Optical fiber sensor for the continuous monitoring of hydrogen in oil // Sensors and Actuators B, 190, p. 982-989 (2014).

35. P. Ngene, R.J. Westerwaal, S. Sachdeva, W. Haije, L.C. P. M. de Smet and B. Dam, Polymer-induced surface modifications of Pd-based thin films leading to improved kinetics in hydrogen sensing and energy storage // Angewandte Chemie Int. Ed. 53, p. 12081-12085 (2014).

36. http://www.adz.de/new-products.html; http://www.variohm.com/pressrelease/detail.php?ai $\mathrm{d}=91 \&$ did=Level-sensor-with-extremely-highcorrosion-resistance; http://www.theengineer.co.uk/supplier-network/ product/variohm-eurosensor-supplies-level-andpoint-sensors-for-applications-such-as-waterways/

37. http://www.plasma.de/en/plasmatechnique/plasmaa pplications.html\#8 encapsulation of PTFE like films by plasma.

38. C. Pannemann, T. Diekmann, U. Hilleringmann et al., PTFE encapsulation for pentacene based organic thin film transistors // Materials Sci. 25(1), p. 95-101 (2007).

39. C. Pannemann, Prozesstechnik für organische Feldeffekt Transistoren: Kontakte, Dielektrika und Oberflächenpassivierungen // Diss. Ph. D., 14/224, Padeborn, 2006.

40. C. Tulkoff, C. Hillman, Understanding nanocoating technology // ESTC, May, Las Vegas, p. 1-52 (2013).

41. A. Brooks, G. Hennighan, S. Woollard, T. von Werne, Plasma polymerization: A versatile and attractive process for conformal coating // IPC APEX EXPO Proc. p. 38-45 (2013).

42. F. Colombo, J. Shah, S. Pendlebury, Protecting electro-optical devices with a fluoropolymer // Patent 20050070196, USA, 2005. 
43. http://appleinsider.com/articles/15/03/05/appleresearching-device-waterproofing-via-vapordeposition-silicone-seals

44. K.P. Gritsenko, Mechanism of PTFE film growth in vacuum // Ukrainian Chem. J. 57(7), p. 782-784 (1991), in Russian.

45. K.P. Gritsenko, Yu.I. Khimchenko, G.V. Lantoukh, Deposition of barrier sublayer of optical carrier by evaporation of polytetrafluoroethylene in vacuum with ionization of degradation products, in book: Optical Recording Information, Ed. V.V. Petrov. Naukova Dumka, Kiev, 1987, p. 8588 (in Russian).

46. V.V. Petrov, K.P. Gritsenko, A.A. Kriuchin, Research on films structure produced by coevaporation metals and PTFE in vacuum // Doklady Akademii Nauk Ukrain. SSR, 2, p. 64-68 (1989), in Russian.

47. K.P. Gritsenko, A.M. Krasovsky, Thin film deposition of polymers by vacuum degradation // Chem. Rev. 103(9), p. 3607-3650 (2003).

48. K.P. Gritsenko, O.P. Dimitriev, V.V. Kisluk, O.M. Getsko, S. Schrader, L. Brehmer, Dye-fluoropolymer nanocomposite film deposition in vacuum // Colloids \& Surf. A: Physchem. Eng. Aspects, 198-200, p. 625-632 (2002).

49. K.P. Grytsenko, S. Schrader, Nanoclusters in polymer matrices prepared by co-deposition from a gas phase // Adv. in Colloid and Interface Sci. 116, p. 263-276 (2005).

50. K.P. Gritsenko, A. Capobianchi, A. Convertino, J. Friedrich, R.D. Schulze, V. Ksensov, S. Schrader, Polymer-metal composite thin film prepared by coevaporation in vacuum, in book: Polymer Surface Modification and Polymer Coatings by Dry Process Technologies, Ed. S. Iwamori, Research Signpost, Kerala, 2005, p. 85-109.

51. K. Grytsenko, O. Navozenko, Yu. Kolomzarov et al., Optical properties of dye-filled polymer films, deposited in vacuum // Data Record., Storage \& Proc. 14(1), p. 3-9 (2012).

52. K.P. Grytsenko, V.F. Machulin, A.O. Ait, A.M. Gorelik, O.I. Kobeleva, T.M. Valova, V.A. Barachevsky, Photochromic films prepared by vacuum co-deposition of polymer and spiropyrans // Optical Memory and Neural Networks, 19(3), p. 254-259 (2010).

53. O.I. Kobeleva, T.M. Valova, A.O. Ait, V.A. Barachevsky, K.P. Grytsenko, V.F. Machulin, M.M. Krayushkin, Photochromic properties of composite films of thermally irreversible diarylethenes and fulgimides in polytetrafluoroethylene matrix // Semiconductor Physics, Quantum Electronics \& Optoelectronics, 14(4), p. 441-444 (2011).

54. K. Grytsenko, S. Schrader, H. Detert, Ultra-stable dye-filled polytetrafluoroethylene thin films // Nanosci. \& Technol. 1(2), p. 1-5 (2014).

55. K. Grytsenko, S. Schrader, O. Tolmachev, Yu. Slominski, V. Barachevsky, O. Kobeleva, Nonconventional properties of dyes in polytetrafluoroethylene matrix // Abstracts of Conf. European Optical Soc., Paris, October 5, 2010, p. 635.

56. K. Grytsenko, T. Doroshenko, Yu. Kolomzarov et al., Growth and optical properties of film of dyes and dye-in-polymer matrix, deposited by evaporation in vacuum // Semiconductor Physics, Quantum Electronics \& Optoelectronics, 13(2), p. 177-179 (2010).

57. I.V. Gorbov, A.A. Kryuchyn, K.P. Grytsenko, D.Yu. Manko, Yu.O. Borodin, High-density data recording via laser thermo-lithography and ion-beam etching // Semiconductor Physics, Quantum Electronics \& Optoelectronics, 17(1), p. 52-55 (2014).

58. V.V. Petrov, A.A. Kryuchin, I.V. Gorbov et al., Photosensitive material for optical recording // Patent 201403344 Ukraine, 2014.

59. K. Grytsenko, Yu. Kolomzarov, P. Lytvyn, Technology of thin films of photosensitive nanocomposite materials and their properties, including pits morphology // Project STCU 5709, Final Report, p. 1-20 (2013).

60. L. Ivanov, K. Grytsenko, Yu. Kolomzarov, O. Tolmachev, Yu. Slominskii, S. Schrader, Organized structures in PTFE film filled with dye and metal nanoparticles and novel research trends // Abstracts of Conference Polycomtrib-2015, June 23-26, 2015, Gomel, p. 29. 